Recherches en didactique des langues et des cultures

Les cahiers de l'Acedle

13-1 | 2016

Interactions langagières et didactique des langues

\title{
Prendre son cours à bras le corps
}

De l'articulation des modalités kinésiques avec la parole

Marion Tellier

\section{OpenEdition}

Journals

Édition électronique

URL : http://journals.openedition.org/rdlc/474

DOI : $10.4000 /$ rdlc. 474

ISSN : $1958-5772$

Éditeur

ACEDLE

Référence électronique

Marion Tellier, "Prendre son cours à bras le corps », Recherches en didactique des langues et des cultures [En ligne], 13-1 | 2016, mis en ligne le 25 juillet 2016, consulté le 01 mai 2019. URL : http:// journals.openedition.org/rdlc/474; DOI : 10.4000/rdlc.474

Ce document a été généré automatiquement le 1 mai 2019.

\section{(c) $(1)$}

Recherches en didactique des langues et des cultures is licensed under a Creative Commons AttributionNonCommercial-NoDerivatives 4.0 International License 


\title{
Prendre son cours à bras le corps
}

De l'articulation des modalités kinésiques avec la parole

\author{
Marion Tellier
}

\section{Introduction}

1 Dans la communication ordinaire, les interactants utilisent spontanément ce que l'on nomme des gestes coverbaux qui ont à la fois un effet facilitateur pour la compréhension du discours verbal qu'ils accompagnent (Beattie \& Shovelton, 1999 ; Holler et al., 2009) et qui constituent une aide à la production langagière (Rauscher et al., 1996). Ces gestes sont construits avec le verbal et peuvent, la plupart du temps, le compléter ou le préciser notamment en transmettant les informations relatives aux aspects visuels (taille, forme, position dans l'espace, déplacement...) (Goldin-Meadow, 2003). McNeill (1985, 1992, 2005) a depuis longtemps argumenté en faveur d'une analyse combinée de ces deux modalités de la parole en montrant qu'elles forment un seul et même système.

2 Qu'en est-il de la communication en classe de langue? Sans aborder ici la parole et la gestuelle de l'apprenant qui mériteraient une analyse approfondie (Gullberg, 2010; McCafferty \& Stam, 2008), on peut reconnaître que la parole de l'enseignant est foncièrement multimodale (Tellier \& Cadet, 2014). L'agir professoral se concrétise à travers plusieurs modalités, "verbales et non verbales" pour reprendre les mots de Cicurel (2011). Dans cet article, nous nous concentrerons sur la modalité gestuelle manuelle même si nous ferons référence à d'autres modalités kinésiques telles que le regard ou les expressions faciales et, parfois même, les mouvements de tête. La multimodalité du discours de l'enseignant de langue n'est plus à démontrer tant en face à face (Tellier, 2008; Azaoui, 2014 a et b, Denizci, 2015) qu’à distance (Cosnier \& Develotte, 2011; Guichon, 2013). Les études portant sur l'analyse des manifestations multimodales de l'enseignant se focalisent en général soit sur la description des gestes utilisés, notamment lors de l'explication lexicale, soit sur les fonctions pédagogiques de ces gestes en montrant comment ils participent à l'agir professoral. 
Cependant, aucune étude à notre connaissance ne s'est focalisée sur les liens entre la parole de l'enseignant et ses gestes au niveau de leur synchronisation d'une part et de leur lien sémantique d'autre part (redondance ou complémentarité). Le présent article portera donc sur ces deux aspects à travers une approche qualitative basée sur un corpus écologique et semi-contrôlé. Dans un premier temps, nous reviendrons sur les fonctions du geste pédagogique en montrant que celui-ci s'articule non seulement avec la parole mais également avec d'autres modalités kinésiques, ce qui nous conduira à parler de corps pédagogique. Puis, nous analyserons la relation sémantique entre le geste et la parole (lorsque le geste est redondant avec celle-ci, lorsqu'il la désambigüise et lorsqu'il la complète). Enfin, nous examinerons finement la synchronie de production entre le geste et la parole en montrant que les deux n'apparaissent pas toujours exactement au même instant.

\section{Fonction du geste pédagogique}

4 Le fonctionnement du geste pédagogique en classe (de langue) est d'une grande complexité. Il ne se limite pas à un simple sous-titrage gestuel de ce que l'enseignant dit mais revêt des formes diverses et occupe différentes fonctions. Nous proposons dans cette partie, d'illustrer cela par l'étude d'un court extrait de corpus ${ }^{1}$ afin d'examiner les gestes manuels mais également d'autres modalités kinésiques (regard, expressions faciales, postures) et vocale (même si l'analyse ne sera qu'effleurée). Il s'agit de l'enregistrement vidéo d'une classe expérimentale mise en place en 2009 à l'université d'Aix-Marseille dans le cadre du master 1 de FLE. Chaque semaine, un groupe de 3 ou 4 étudiants (futurs enseignants) devait concevoir une séance de $2 \mathrm{~h}$ pour des apprenants de FLE de niveaux variés. Les apprenants étaient des volontaires et n'étaient pas nécessairement les mêmes d'une semaine à l'autre. Dans cette séance, Philippe, un des étudiants du groupe, commence par introduire le sujet du jour (la grève en France) par une devinette. Philippe a déjà enseigné le FLE en Angleterre et en Amérique du Sud pendant 2 ans.

Tableau 1 - Corpus classe expérimentale - Séquence « la grève _ anticipation » Cours de FLE de jeunes adultes (niveau A2-B1)

\begin{tabular}{|l|l|l|}
\hline 1 & E & $\begin{array}{l}\text { alors (360) la question j(e) } \\
\text { vais vous poser de } \\
\text { plusieurs fois de } \\
\text { différentes manières c'est } \\
\text { très simple (1540) quel est } \\
\text { (470) le sport national } \\
\text { français (410) }\end{array}$ \\
\hline 3 & A1 & la pétanque (340) \\
\hline 5 & E & $\begin{array}{l}\text { la pétanque (230) hum peut- } \\
\text { etre (420) une autre idée }\end{array}$ \\
\hline 6 & A1 & XxX (420) football (340) \\
\hline 7 & E & le football hum \\
\hline
\end{tabular}




\begin{tabular}{|c|c|c|}
\hline 8 & $\mathrm{~A} 1$ & rugby (260) \\
\hline 9 & $\mathrm{E}$ & le rugby ouais \\
\hline 10 & A2 & oui oui oui (rires) (490) \\
\hline 11 & $\mathrm{E}$ & des idées (840) Ikari (1410) \\
\hline 12 & A1 & foot (210) \\
\hline $\begin{array}{l}13 \\
14 \\
15 \\
16\end{array}$ & $\mathrm{E}$ & $\begin{array}{l}\text { le foot hum }(1230) \text { OK alors } j \\
\text { (e) vous donne un deuxième } \\
\text { indice (2020) grâce à ce } \\
\text { sport (790) on peut jouer } \\
\text { (390) à plusieurs millions } \\
\text { de personnes plusieurs } \\
\text { millions de personnes dans } \\
\text { une même équipe (1340) } \\
\text { alors de quoi je parle }\end{array}$ \\
\hline 17 & $\mathrm{~A} 2$ & XX (400) la politique \\
\hline 18 & A1 & jeux vidéo \\
\hline 19 & $\begin{array}{l}\text { A1 } \\
\text { A } 2\end{array}$ & $\underline{\text { (rires) }}$ \\
\hline 20 & $\mathrm{E}$ & $\begin{array}{l}\text { ah la politique ah c'est pas } \\
\text { bête (310) }\end{array}$ \\
\hline 21 & A1 & (rires) \\
\hline 22 & $\mathrm{E}$ & une idée \\
\hline 23 & A2 & de la grève (260) \\
\hline $\begin{array}{l}24 \\
25\end{array}$ & $\mathrm{E}$ & $\begin{array}{l}\text { la grève c'est une très c'est } \\
\text { une c'est une excellente idée } \\
\text { quelqu'un a une autre idée }\end{array}$ \\
\hline 26 & $\begin{array}{l}\mathrm{A} 1 \\
\mathrm{~A} 2\end{array}$ & (rires) \\
\hline 27 & A1 & $\begin{array}{l}\text { je p- (en)fin c'est pas un sport } \\
\text { enfin un jeu vidéo }\end{array}$ \\
\hline 28 & $\mathrm{E}$ & $\begin{array}{l}\text { un jeu vidéo ouais c'est vrai } \\
\text { ouais (550) OK (420) }\end{array}$ \\
\hline
\end{tabular}




\begin{tabular}{|c|c|c|}
\hline 29 & A1 & $\mathrm{XX}$ \\
\hline 30 & A2 & un sport (240) \\
\hline 31 & A1 & XXX (280) \\
\hline 32 & A1 & casino $(220)$ \\
\hline $\begin{array}{l}33 \\
34 \\
35\end{array}$ & $\mathrm{E}$ & $\begin{array}{l}\text { le casino OK pourquoi pas } \\
\text { alors }(420) \text { grâce j(e) donne } \\
\text { un autre indice }(320) \text { grâce } \\
(270) \text { à }(450) \text { cette euh cette } \\
\text { activité ou ce sport (620) on } \\
\text { a gagné (640) le droit de } \\
\text { partir en vacances (290) en } \\
1937 \text { en France }\end{array}$ \\
\hline 36 & A1 & au loto (580) \\
\hline 37 & $\mathrm{E}$ & le loto \\
\hline 38 & A1_A2 & $\underline{\text { (rires) }}$ \\
\hline 39 & E & $\begin{array}{l}\text { oui ah c'est vrai c'est vrai } \\
\underline{\text { aussi (rires) c'est vrai qu'est- }} \\
\underline{\text { ce que tu dis }}\end{array}$ \\
\hline 40 & A2 & non (880) \\
\hline 41 & E & quelqu'un a d'autre idée \\
\hline
\end{tabular}

5 Dans la transcription, on voit que l'enseignant commence son anticipation par poser une question «quel est (470) le sport national français (410)» (lignes 1-3) puis relance la discussion à différents moments par des indices (lignes 14-16 et 34-35). On retrouve d'ailleurs dans cette structuration les traces de la planification du cours de Philippe. À tout moment, les apprenants proposent différentes réponses à la question que l'enseignant valide en les reprenant (il n'est pas question ici de trouver la bonne réponse mais de faire parler les apprenants et de les intéresser au sujet par l'énigme mise en place). L'enseignant reprend les énoncés des apprenants en écho (Cicurel, 1990 : 53) ou produit des hétérocorrections autodéclenchées, en ajoutant parfois l'article quand il a été omis par l'apprenant (lignes 7, 9, 13, 33, 37). Les reprises des réponses des apprenants sont accompagnées de formules d'appréciation très variées (indiquées en gras dans les exemples suivants) : « la pétanque hum peut-être » (ligne 4), « le football hum » (ligne 7), « le rugby ouais » (ligne 9), « le foot hum » (ligne 13), " ah la politique ah c'est pas bête » (ligne 20), « la grève c'est une très c'est une c'est une excellente idée » (ligne 24), « un jeu vidéo ouais c'est vrai ouais » (ligne 28), «le casino OK pourquoi pas » (ligne 33). L'analyse de la transcription des échanges verbaux permet de bien prendre en compte ce 
qui se joue dans la classe mais cela demeure limité par le fait de ne pas avoir accès aux indices posturo-mimo-gestuels, comme l'exprime Mondada :

La prise en compte de la mobilisation complexe, mais ordonnée et systématique, de ressources gestuelles et corporelles en interaction ne peut laisser intactes les descriptions linguistiques classiques, exclusivement basées sur le verbal et souvent en isolation par rapport à des usages situés. (2012:129).

En effet, en analysant les gestes manuels, le regard, les mouvements de têtes et le visage de l'enseignant, on peut observer la façon dont il orchestre les échanges multimodalement ${ }^{2}$ et quelles fonctions, l'usage du corps associé à la parole, remplit dans la classe de langue. L'analyse de la gestuelle pédagogique a permis de mettre au jour trois grandes catégories fonctionnelles du geste (Tellier, 2006 et 2008): les gestes d'information, les gestes d'évaluation et les gestes d'animation. Ce découpage rappelle, bien évidemment, les rôles de l'enseignant définis par Dabène (1984). Il semble tout à fait pertinent de réutiliser cette catégorisation fonctionnelle, originellement bâtie sur l'analyse d'interactions verbales, pour l'appliquer à l'analyse des phénomènes gestuels d'autant plus que Dabène prend le soin de préciser «qu'un bon nombre d'opérations peuvent se traduire par des manifestations non verbales » (1984: 43). Bien qu'ancienne, cette typologie demeure tout à fait pertinente pour l'analyse de l'action enseignante. Nous reprenons dans les pages suivantes cette typologie, déjà développée ailleurs (Tellier, 2008) mais en approfondissant l'analyse en y intégrant d'autres modalités kinésiques et, dans une moindre mesure, la prosodie.

\section{La fonction d'information}

7 Parmi les gestes d'information, on trouve des gestes d'information grammaticale qui servent à transmettre des données relatives à la morphosyntaxe et à la temporalité, des gestes d'information lexicale pour illustrer un mot ou une idée du discours oral de l'enseignant et des gestes d'information phonologique et phonétique créés pour aider les apprenants à maitriser la prononciation et la prosodie (Tellier, 2008). La fonction d'information du geste (et du corps en général), notamment lors de l'explication lexicale a été beaucoup étudiée (Kellerman, 1992, Allen, 2000, Pavelin, 2002, Lazaraton, 2004, Holt, 2014, entre autres). Le geste peut être utilisé pour faciliter l'explication d'un terme qui pose problème ou pour désambiguïser deux expressions (voir l'exemple de " petit bœuf » et «petit morceau de bœuf» plus loin dans cet article). Cependant, le geste n'intervient pas uniquement lorsque l'incompréhension a eu lieu chez l'apprenant et que l'enseignant doit mettre en place une séquence de réparation. Au contraire, on remarque dans le discours multimodal du professeur, une anticipation des difficultés potentielles de compréhension. En somme, il projette sur l'apprenant des connaissances, des lacunes et des besoins.

Le geste est produit par l'enseignant pour illustrer un mot ou une idée de son discours oral. Il peut choisir d'illustrer un mot plutôt qu'un autre pour deux raisons principales (les deux pouvant être combinées): (1) parce qu'il juge ce terme particulièrement important pour comprendre le sens global de la phrase (c'est le pivot) (2) parce qu'il suppose que ce mot est inconnu de l'apprenant et va lui poser problème. (Tellier, $2008:$ 43)

8 Dans l'extrait de corpus "la grèveanticipation », les trois séquences, durant lesquelles l'enseignant pose la question principale et donne ensuite les indices (en gras dans la transcription), montrent bien l'usage combiné des modalités verbales, vocales et 
kinésiques ${ }^{3}$. La question posée ( « quel est le sport national français ») est mise en forme grâce à l'usage de différentes modalités concomitantes (figure 1). Au niveau prosodique, sans faire d'analyse poussée, on remarque une pause de $470 \mathrm{~ms}$ juste avant le mot clé «sport national » et une pause plus courte de $120 \mathrm{~ms}$ avant le mot «français ». Le jeu des intonations montantes sur « quel est » et « national » laisse en suspens les mots et montre que la question n'est pas terminée. L'ensemble facilite la segmentation de la châne parlée pour l'apprenant.

Figure 1 - Mise en évidence de concepts clés (1)

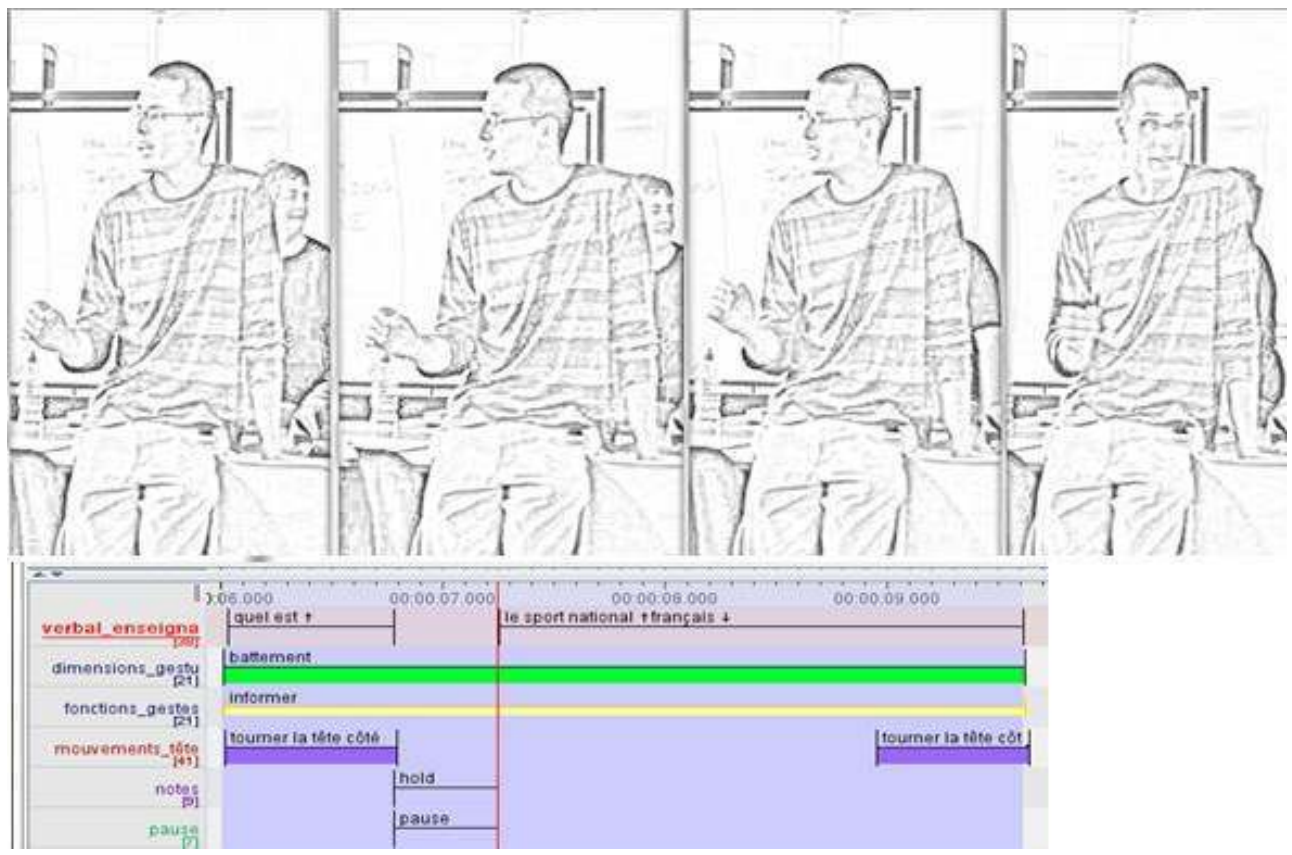

[quel est (0.47) le sport national (0.12) français] $]^{4}$

Pendant la durée de la question, un ensemble de battements ${ }^{5}$, main fermée, ponctue chaque mot de la phrase. Pendant la pause entre «quel est » et « le sport», le geste reste lui aussi suspendu dans un hold (voir la capture d'écran du logiciel Elan, figure $1^{7}$ ).

Enfin, le regard balaie l'ensemble de la classe : la tête de l'enseignant se déplace d'abord vers la droite puis vers la gauche et la main accompagne le mouvement. Ce professeur a donc à cœur de bien regarder l'ensemble de ses interlocuteurs et d'orienter les gestes dans leur direction pour les rendre visibles par tous. Ce phénomène d'orientation du geste vers l'interlocuteur a déjà été mis en évidence par Özyürek (2002) dans des dyades ou trio, en contexte semicontrôlé et, il est intéressant de remarquer la même chose, en situation écologique, dans cette classe où l'enseignant est coénonciateur de plusieurs apprenants à la fois pour reprendre l'expression d'Azaoui (2014a et b). 
Figure 2 - Mise en évidence de concepts clés (2)

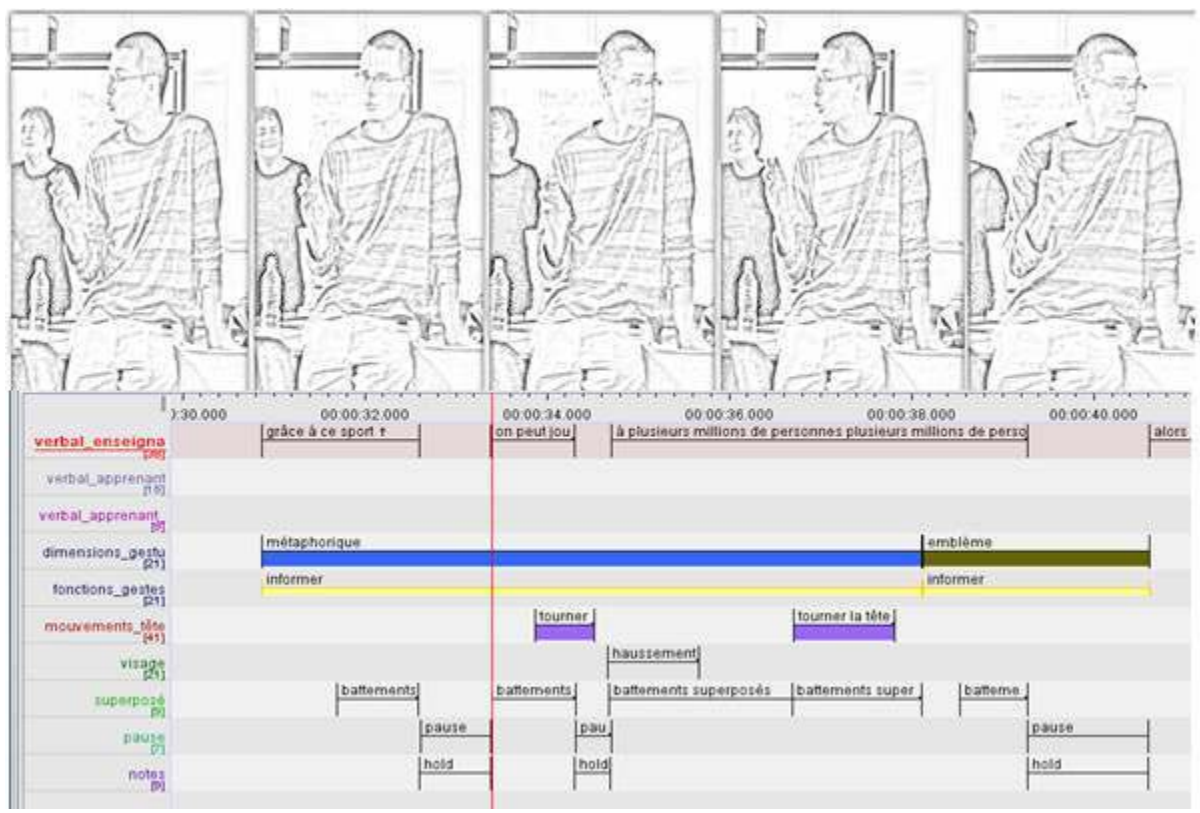

[grâce à ce sport (0.79) on peut jouer (0.39) à plusieurs millions de personnes plusieurs millions de personnes dans une même équipe (1.34)]

Dans le premier indice donné (lignes 13 à 16) pour relancer la séquence d'anticipation, Philippe annonce "grâce à ce sport (790) on peut jouer (390) à plusieurs millions de personnes plusieurs millions de personnes ». Pendant cette séquence, il produit un geste métaphorique $^{8}$ (main droite crispée comme si elle tenait quelque chose, paume vers l'extérieur) (figure 2). Ce geste est renforcé par de nombreux battements superposés sur le geste métaphorique qui scandent la phrase pour insister sur «plusieurs millions » qui est un indice essentiel dans son discours. À la fin de l'énoncé, « dans une même équipe (ligne 34) », l'enseignant produit un emblème ${ }^{9}$ avec l'index dressé pour illustrer « une ».

Il contraste ainsi les deux éléments clés et paradoxaux de sa phrase "plusieurs millions de personnes » et « une même équipe » à grands renforts de gestes. Dans les pauses qui ponctuent le discours de Philippe, les gestes sont maintenus dans des holds comme dans l'extrait précédent, marquant ainsi les mots-clés (figure 2). Une fois encore, comme on peut le voir sur la série de clichés et sur la capture du fichier d'annotation, les mouvements de tête d'un côté et de l'autre montrent que son regard balaie l'ensemble des apprenants.

\section{La fonction d'évaluation}

Dans la catégorie geste d'évaluation sont classés les gestes et autres éléments kinésiques utilisés pour féliciter, approuver et signaler une erreur. Ceux utilisés pour féliciter/ approuver apparaissent davantage à la fin de l'intervention de l'apprenant, ce qui permet à l'enseignant de marquer la fin de la réponse en évaluant positivement ou pendant la production (par exemple en acquiesçant et en souriant) pour encourager l'apprenant à poursuivre. Lorsque l'enseignant veut signaler une erreur dans l'énoncé oral d'un apprenant sans l'interrompre, il a tendance à le faire de manière kinésique (et donc silencieuse) souvent pour indiquer un problème dans la réponse de l'élève et non pour lui 
donner la bonne réponse. L'apprenant, sachant où se trouve la partie erronée, n'a plus qu'à la corriger et peut ainsi réfléchir sur ses erreurs (Tellier, 2008). Cette fonction d'évaluation, notamment dans la classe de langue, a fait l'objet de quelques recherches principalement descriptives (Martina, 1991; Mackey et al., 2007; Faraco, 2008). Sans en faire une analyse exhaustive ici, les évaluations que Philippe produit suite aux énoncés des apprenants sont constituées de différentes modalités utilisées conjointement.

Figure 3 - Évaluation (1)

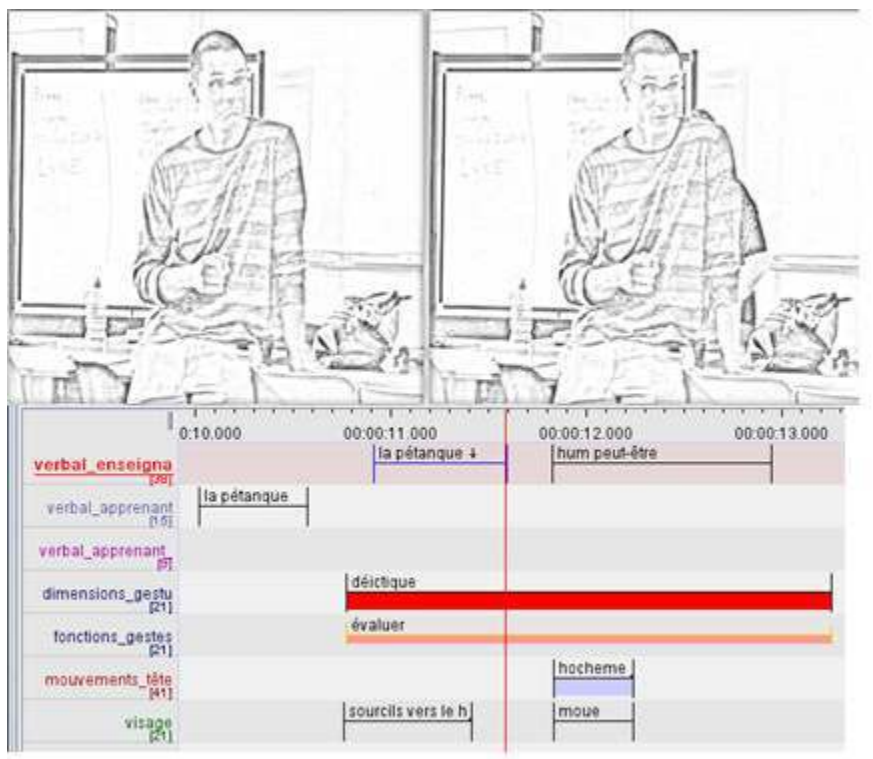

[la pétanque (0.23) hum peut-être]

Lorsqu'un premier apprenant propose la réponse «la pétanque », l'enseignant pointe en direction du locuteur pour le singulariser dans la classe tout en reprenant la réponse, ce qui la valide comme acceptable. Il produit aussi un haussement de sourcils au début du geste déictique (de pointage) qui montre l'intérêt qu'il porte à la réponse. Toujours en pointant vers l'apprenant, il hoche la tête et produit une moue et dit « hum peut-être » (figure 3). De la même manière, lorsqu'un apprenant propose «le football », il reprend cette proposition, tout en pointant le doigt vers celui qui a fourni la réponse, puis penche la tête sur le côté et produit un emblème (main ouverte pivotant légèrement sur le côté que l'on peut utiliser avec l'expression "pourquoi pas»). Il accompagne ce geste d'une moue (figure 4) et d'un signal vocal de feedback « hum ». 
Figure 4 - Évaluation (2)

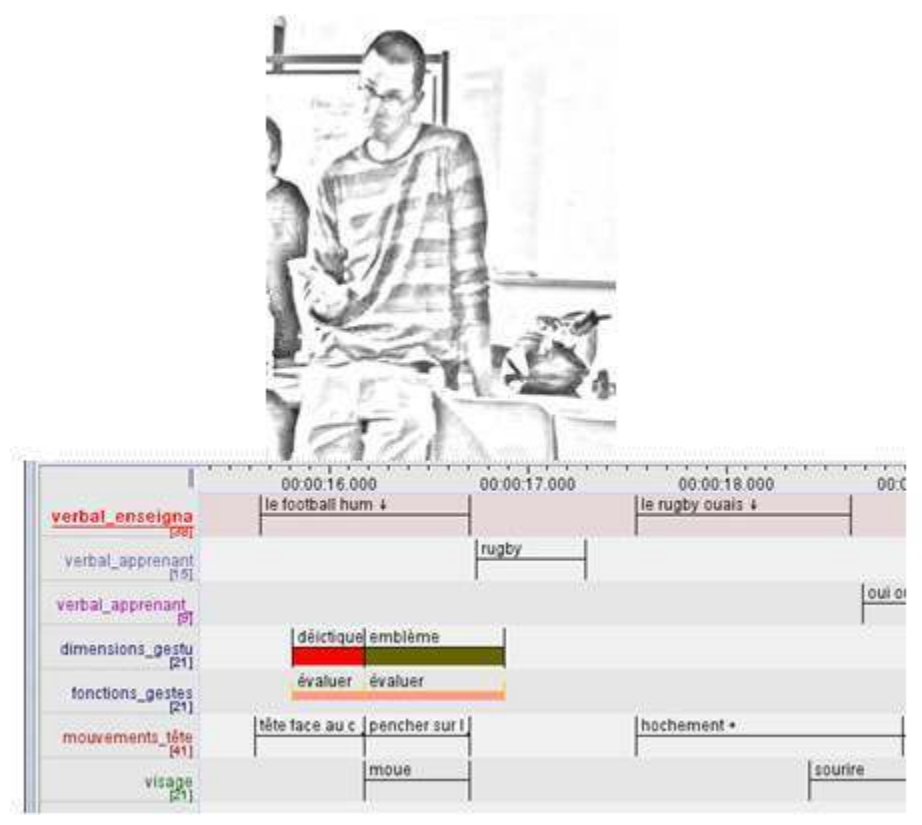

le [football hum] ... le rugby ouais

\section{La fonction d'animation}

La troisième grande catégorie fonctionnelle de geste pédagogique est le geste d'animation qui englobe à la fois les gestes de gestion de classe (changement d'activité, démarrage et clôture d'activité, placement des apprenants/du matériel, punir/gronder/ faire taire, donner des consignes) et de la gestion des interactions et de la participation (réguler les débits/le volume sonore, faire répéter, étayer, interroger, donner la parole) (Tellier, 2008). C'est une fonction relativement peu étudiée dans l'analyse de la gestuelle de l'enseignant et quand elle est mentionnée, elle fait rarement l'objet d'une analyse minutieuse (Grant \& Grant Hennings, 1971, Barnett, 1983) à l'exception des travaux d'Azaoui (2014a et b) qui prend d'ailleurs en compte différents signaux kinésiques. L'extrait de corpus de la classe de Philippe présente peu d'exemples de la fonction d'animation. Il utilise cette fonction principalement dans la gestion des interactions en pointant l'index vers un apprenant et en le regardant pour lui donner la parole comme on peut le voir dans l'exemple de la figure 5.

Bien entendu, lorsque l'on classe les gestes des apprenants selon les fonctions pédagogiques qu'ils remplissent, on constate souvent qu'un même geste peut remplir deux fonctions simultanément. Ainsi, lorsqu'un enseignant reprend la réponse d'un apprenant en l'illustrant d'un geste, il valide la réponse (fonction d'évaluation) tout en marquant gestuellement le mot-clé pour faciliter la compréhension des autres apprenants (fonction d'information). 
Figure 5 - Animation : distribution de la parole

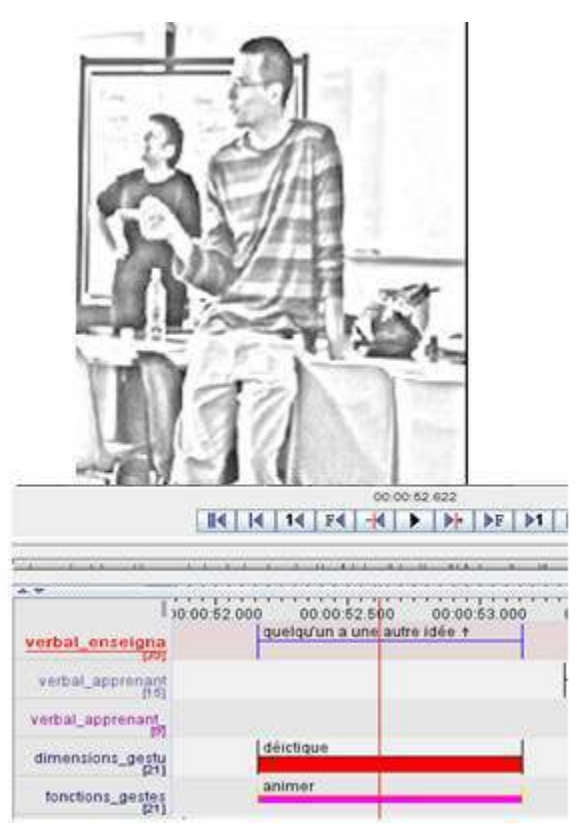

[quelqu'un a une autre idée]

17 Le découpage en fonction pédagogique des modalités kinésiques utilisées par l'enseignant apporte un éclairage plus précis de l'agir professoral. Cependant, une analyse plus fine du lien geste/parole permet d'aller plus loin dans la compréhension du discours pédagogique multimodal.

\section{Les liens sémantiques}

18 Afin de mettre en évidence les liens sémantiques qui unissent la production du geste et de la parole, nous utiliserons ici un autre extrait issu du même corpus de classe expérimentale. Dans l'extrait ci-dessous, Virginie, jeune enseignante de FLE, étudie avec ses apprenants un dialogue se déroulant au restaurant. Un des personnages du dialogue commande de la bavette. Une apprenante demande alors la signification de ce mot. L'extrait ci-dessous montre la réponse verbale de Virginie (Vir) ${ }^{10}$.

Tableau 2 - Corpus classe expérimentale - Séquence " la bavette » Cours de FLE de jeunes adultes (niveau A2-B1)

\begin{tabular}{|c|c|c|}
\hline 1 & A1 & la bavette (330) \\
\hline 2 & Vir & $\begin{array}{l}\text { bavette (330) alors la bavette on } \\
\text { entend }\end{array}$ \\
\hline 3 & A1 & c'est (450) \\
\hline 4 & A2 & une type de viande (620) \\
\hline
\end{tabular}




\begin{tabular}{|c|c|c|}
\hline 5 & Vir & $\begin{array}{l}\text { ouais (850) c'est un type de } \\
\text { viande tu as une idée }\end{array}$ \\
\hline 6 & A2 & bœuf bœuf mais petit (1210) \\
\hline 7 & Vir & $\begin{array}{l}\text { petit bœuf ah c'est petit bœuf } \\
\text { [petit morceau de bœuf -1-] ou } \\
\text { [petit bœuf -2-] }\end{array}$ \\
\hline 8 & A2 & non petit morceau \\
\hline $\begin{array}{l}9 \\
10\end{array}$ & Vir & $\begin{array}{l}\text { petit (rires) alors c'est pas [un } \\
\text { bébé bœuf (370)] [c'est un } \\
\text { morceau de bœuf mais oui c'est } \\
\text { des petits morceaux de y a pas } \\
\text { d'os (210) dans la bavette] }\end{array}$ \\
\hline 11 & A1 & $\mathrm{Ah}$ \\
\hline $\begin{array}{l}12 \\
13\end{array}$ & Vir & $\begin{array}{l}\text { [et c'est des tranches-3-] [(330) } \\
\text { fines }(650)-4-] \text { [et comment on } \\
\text { les cuit (660) vous savez le mot } \\
-5-\text { - }\end{array}$ \\
\hline
\end{tabular}

L'enseignante commence par solliciter les apprenants à la ligne 2 et très rapidement une apprenante propose une réponse "une type de viande " (ligne 4) que l'enseignante demande de préciser (ligne 5). Suit alors une réponse ambigüe de l'apprenante «bœuf bœuf mais petit » (ligne 6). S'agit-il du petit du bœuf (le veau) ou bien d'un petit morceau de bœuf? L'interaction des lignes 7 à 10 a pour objectif de désambigüiser ceci. L'enseignante précise alors la définition de bavette «c'est des tranches [(330) fines (650)]» (ligne 12) puis relance la discussion par une question « et comment on les cuit (660) vous savez le mot " (lignes 12-13). Dans cette interaction de classe assez classique autour d'une explication lexicale, on retrouve les rouages fondamentaux de la parole enseignante et de l'habitus didactique (Cicurel, 1990). Mais ce que cette transcription ne montre pas c'est la façon dont le corps de l'enseignante participe à cette action. En effet, à partir de la ligne 7, tout le discours de Virginie est accompagné de gestes ( 9 en tout). Sans les analyser de manière exhaustive, on peut en détailler cinq qui ont des liens différents avec la parole.

\section{Quand le geste désambiguïse la parole}

Les deux premiers gestes (figure 6) permettent de désambigüiser la réponse de l'apprenante. Le premier geste illustre le "petit morceau ", il est produit avec les deux mains arquées comme si elles tenaient quelque chose (ce contenu invisible a la taille et la forme d'un steak). Le second fait référence au «bébé bœuf» (l'expression est utilisée ligne 9 avec le même geste). Un simple geste de la main, paume à plat dirigée vers le sol, indique un référent de petite taille. Dans les deux cas, il s'agit d'illustrer le même adjectif 
« petit » mais selon que l'on fait référence à un morceau de viande ou à un veau, la valeur de « petit » est différente, ce que seul le geste permet ici de clarifier.

Figure 6 - Désambiguïsation par le geste

(1)
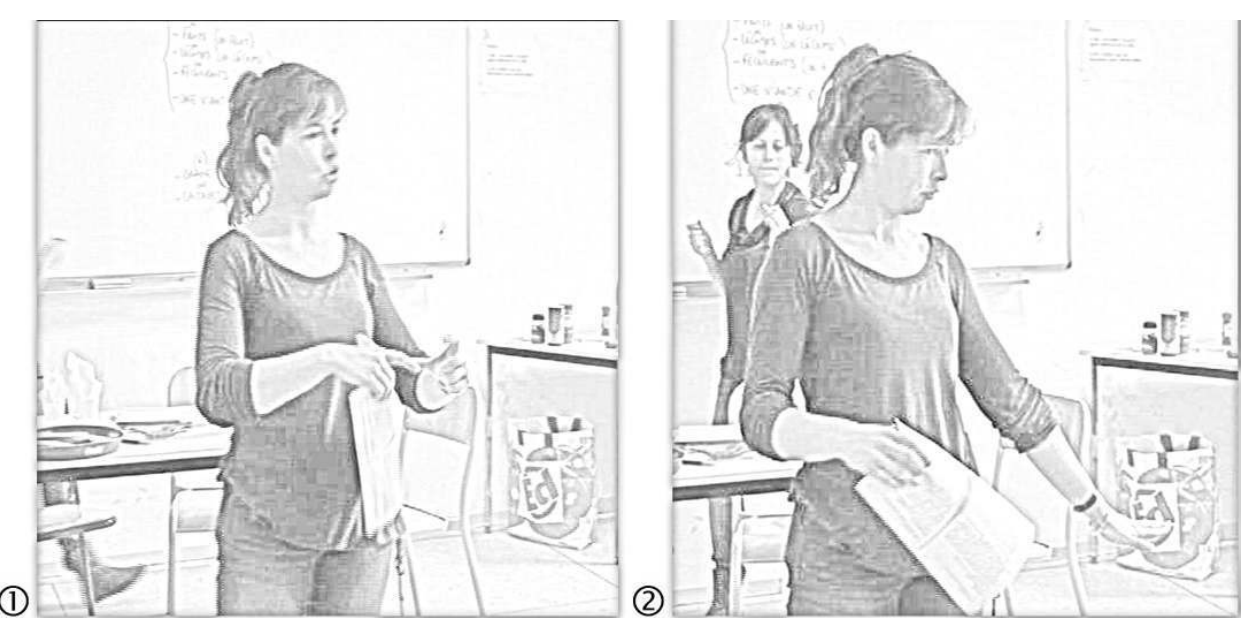

[petit morceau de bœuf -7-] ou [petit bœuf -2-]

\section{Quand le geste sous-titre la parole}

21 Un peu plus loin dans l'explication, elle précise que la bavette est caractérisée par des «tranches fines », ces deux termes sont également illustrés par des gestes (figure 7). Le premier -3- est composé de l'index et du pouce posés parallèlement, l'écart entre ces deux doigts indique quelque chose de peu épais. Ce geste accompagne le mot «tranche » mais comprend déjà l'idée de "fines ». Lorsque cet adjectif apparait dans le discours, il est illustré par un geste des deux mains-4-, identique en configuration manuelle au geste -3mais dynamique. En effet, dans ce geste, les mains réunies ensemble au départ s'écartent doucement et sur une ligne imaginaire afin de dessiner une tranche fine, comme le montrent les flèches sur la photo. Dans ce cas, le geste double en quelque sorte la parole pour en faciliter la compréhension. Geste et parole sont donc dans une relation de redondance. 

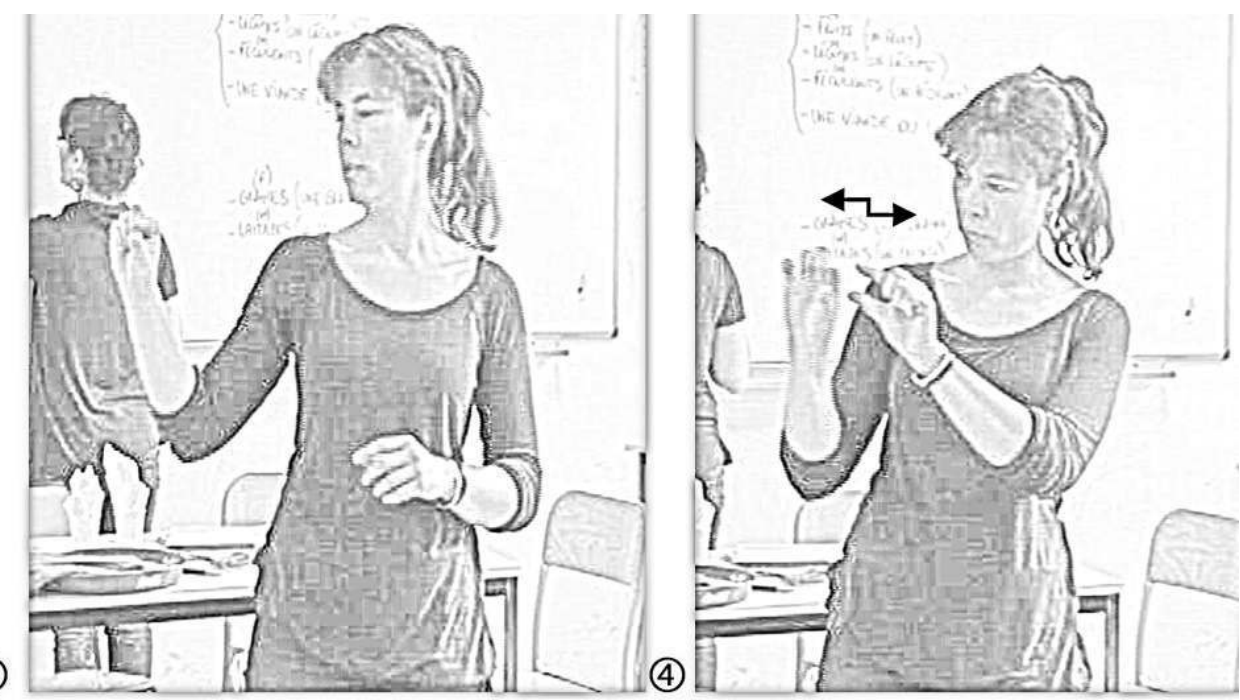

[et c'est des tranches-3-] [(0.33) fines (0.65) -4-]

\section{Quand le geste complète la parole}

Enfin, une fois cette explication lexicale terminée, l'enseignante relance la discussion (dans un but pédagogique puisqu'elle vérifie la compréhension du texte et souhaite faire réemployer du lexique). Elle pose la question « et comment on les cuit (0.66) vous savez le mot ». Si on ne voit pas le geste qui accompagne cette question, il est assez difficile de savoir quelle réponse Virginie attend. Mais si l'on examine ses mains (figure 8), on voit qu'elle bascule plusieurs fois sa main droite à plat pour orienter successivement sa paume vers le ciel puis vers le sol.

Ce geste indique qu'elle attend la réponse "grillée ». Le geste et la parole sont donc ici dans une relation de complémentarité car ils ne disent pas la même chose et se complètent. Ce qui se passe dans cet exemple est très fréquent dans le discours pédagogique, l'enseignante pose une question mais attend une réponse bien précise que son geste indique. Ce qu'elle attend donc, c'est une mise en mots par les apprenants et dans la langue cible de ce qu'elle montre gestuellement. 


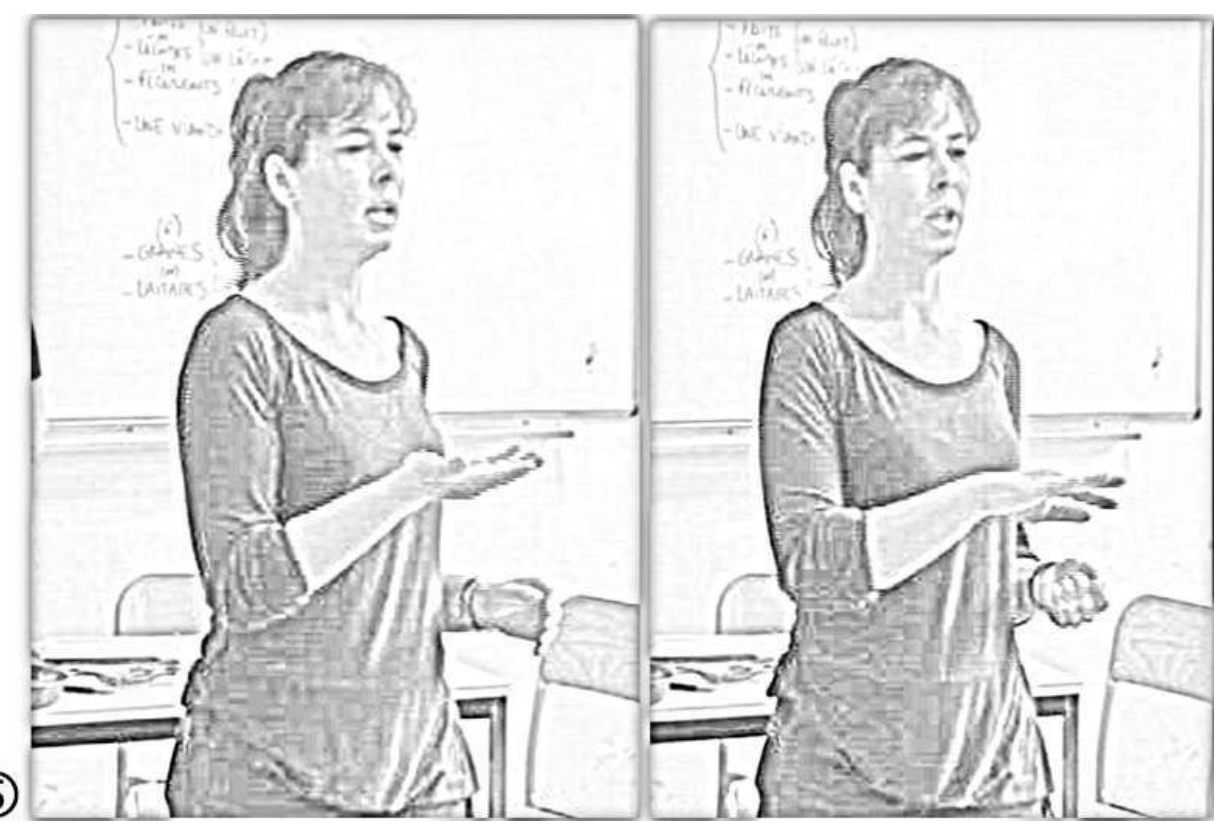

[et comment on les cuit (0.66) vous savez le mot -5-]

\section{Synchronisation gestes, pauses et parole}

Pour finir, nous proposons ici une analyse fine des moments de production du geste et de la parole pour montrer, notamment, comment les pauses structurent le discours didactique, à la fois dans un contexte de classe (contexte écologique) et dans un contexte de tâche imposée (corpus semicontrôlé).

\section{Les gestes dans les pauses du discours pédagogique en classe}

L'exemple de l'explication de «la bavette » par l'enseignante Virginie montre comment elle utilise conjointement gestes et parole pour construire son explication. Un autre élément qui n'a pas été abordé ici est l'usage des pauses dans le discours de l'enseignant (que l'on a pu voir également dans le discours de l'enseignant Philippe, dans le cours sur la grève). Les pauses du discours oral relèvent de la prosodie. Dans le discours didactique, les pauses sont souvent utilisées avec une visée pédagogique. Ainsi dans l'exemple de Virginie, lorsqu'elle précise que la bavette est composée de «tranches fines », elle produit un geste pour illustrer « fines " qui commence avant que cet adjectif ne soit produit et se termine après l'émission de ce mot (figure 7). De la même façon, lorsqu'elle cherche à faire produire le mot "grillé » aux apprenants, elle dit «et comment on les cuit (660) vous savez le mot» tout en produisant un geste qui se poursuit pendant la pause de 660ms au milieu de son énoncé (figure 8). Il y a ici deux usages pédagogiques des gestes produits dans les pauses de son discours. Dans le premier cas, elle illustre des mots-clés en produisant un geste avant et après l'émission du mot. La pause permet à l'apprenant de bien segmenter la phrase en isolant le terme important «fines» tandis que le geste introduit le concept comme pour en faciliter la compréhension et le suit pour marquer le mot. Dans le deuxième cas, le geste sert de support non pas pour faire comprendre mais 
pour faire produire. Lorsqu'elle dit « et comment on les cuit (660) vous savez le mot » si on ne voit pas son geste, on ne peut pas savoir quelle réponse elle attend. Le geste guide l'apprenant, il donne un indice de ce qui est attendu.

\section{Les gestes dans les pauses du discours exolingue en tandem}

Dans une étude de Tellier \& Stam (2012) sur les gestes des futurs enseignants de FLE, il était demandé à des étudiants de master 1 de FLE de faire deviner des mots tirés au sort à un partenaire francophone natif et à un apprenant de FLE (B1-B2). Dans l'exemple suivant, Carole, étudiante en master FLE fait deviner le mot «râpé » à une partenaire apprenante de FLE. Le script de cette séquence est reproduit ici :

Tableau 3 - Corpus GTT_Car_RÂPÉ_NN

\begin{tabular}{|c|c|c|}
\hline $\begin{array}{l}1 \\
2 \\
3 \\
4\end{array}$ & Car. & $\begin{array}{l}\text { quand tu prends du fromage } \\
\text { (790) tu sais du de l'emmental } \\
\text { (580) pour manger avec les pâtes } \\
\text { (470) euh il gros comm(e) ça } \\
\text { (200) et puis tu veux le mettre } \\
\text { sur tes pâtes alors tu le passes } \\
\text { (660) pour qu'il devienne un } \\
\text { (400) en p(e)tits fils (600) est-c } \\
\text { (e) que tu connais euh (250) }\end{array}$ \\
\hline 5 & $\mathrm{NN}$ & oui oui je vois \\
\hline 6 & Car. & $\begin{array}{l}\text { on dit qu(e) le fromage est } \\
(520)\end{array}$ \\
\hline 7 & $\mathrm{NN}$ & Râpé \\
\hline 8 & Car. & Voilà \\
\hline
\end{tabular}

On constate dans le discours verbal de la future enseignante, l'utilisation d'un certain nombre de stratégies verbales, notamment la contextualisation et la description, mais on voit aussi un grand nombre de pauses, indiquées en $m s$ entre parenthèses. Ces pauses dans le discours semblent avoir pour objectif de marquer les mots-clés de l'explication et ainsi de faciliter chez la partenaire non native, la segmentation du discours et le repérage des termes importants. Les pauses apparaissent ainsi après "fromage ", " emmental ", "pâtes », etc. Ce qui est remarquable dans cet extrait, c'est que la gestuelle est constante, malgré le nombre de pauses dans le discours oral. Comme on peut les voir dans les trois extraits ci-dessous, le positionnement de la pause par rapport aux mots-clés et la forme du geste produit dans la pause permet de mettre en évidence et d'illustrer les éléments de l'explication que la future enseignante juge pertinents. Dans l'exemple 1 (figure 9), Carole produit un geste iconique à deux mains comme si elle tenait un morceau de fromage de forme rectangulaire. Ce geste commence lorsqu'elle dit «tu prends du fromage » et se termine juste avant qu'elle commence l'énoncé suivant «tu sais de l'emmental ». Entre les deux énoncés, elle effectue une pause de $790 \mathrm{~ms}$ pendant laquelle le geste est 
maintenu. La pause et le geste permettent donc d'insister sur le mot « fromage ». Pendant cette explication, elle regarde sa partenaire.

Figure 9 - Geste produit dans les pauses du discours - Exemple 1

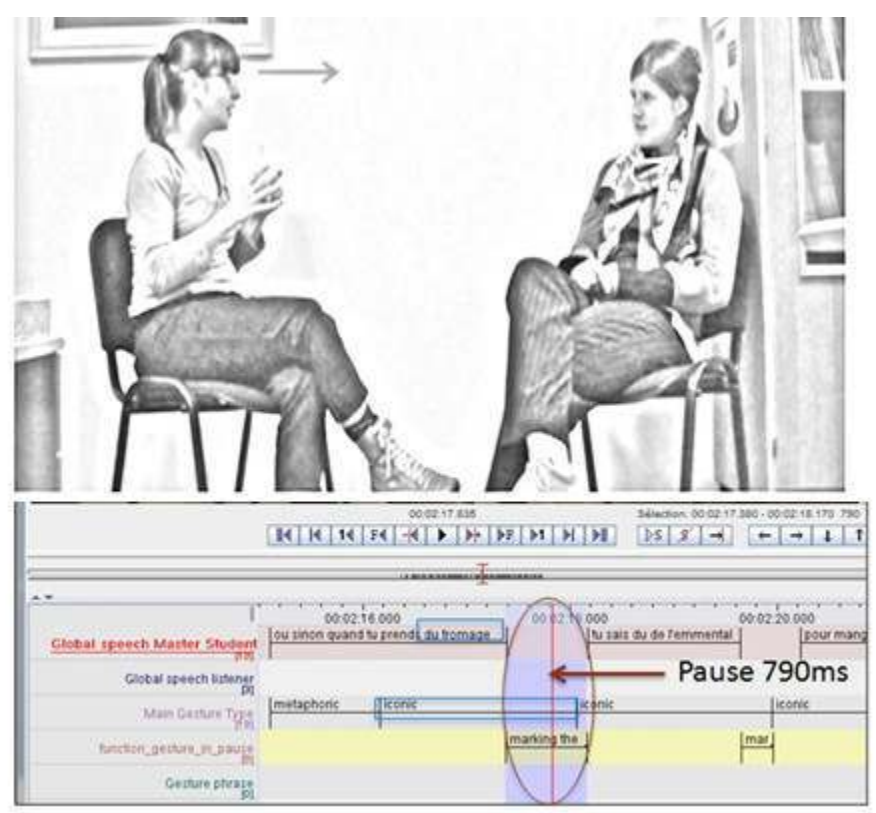

[ou sinon quand tu prends du fromage (0.79) tu sais de l'emmental]

Elle poursuit son explication en indiquant « pour manger avec les pâtes ». À la fin de cet énoncé, elle commence à produire un geste (figure 10) qui ressemble légèrement au geste qu'elle avait produit pour illustrer le mot fromage mais cette fois, il s'agit d'indiquer la taille. Ce geste est accompagné de l'énoncé « euh il gros comm(e) ça ». La relation entre le geste et la parole est ici complémentaire car chacun apporte des informations différentes et complète l'autre (la taille n'est indiquée que par le geste) alors que dans l'exemple 1, il s'agissait d'une relation de redondance dans la mesure où le geste et la parole véhiculaient la même information. Entre les deux énoncés, elle marque une pause de $470 \mathrm{~ms}$ pendant laquelle le geste commence. Ainsi, au lieu de marquer le mot-clé par un geste dans une pause après l'émission du mot, elle illustre la taille avant de prononcer " gros comme ça ». En quelque sorte, elle anticipe sur le contenu de son énoncé et permet peut-être ainsi à sa partenaire de mieux comprendre ce qui va suivre. 
Figure 10 - Geste produit dans les pauses du discours - Exemple 2

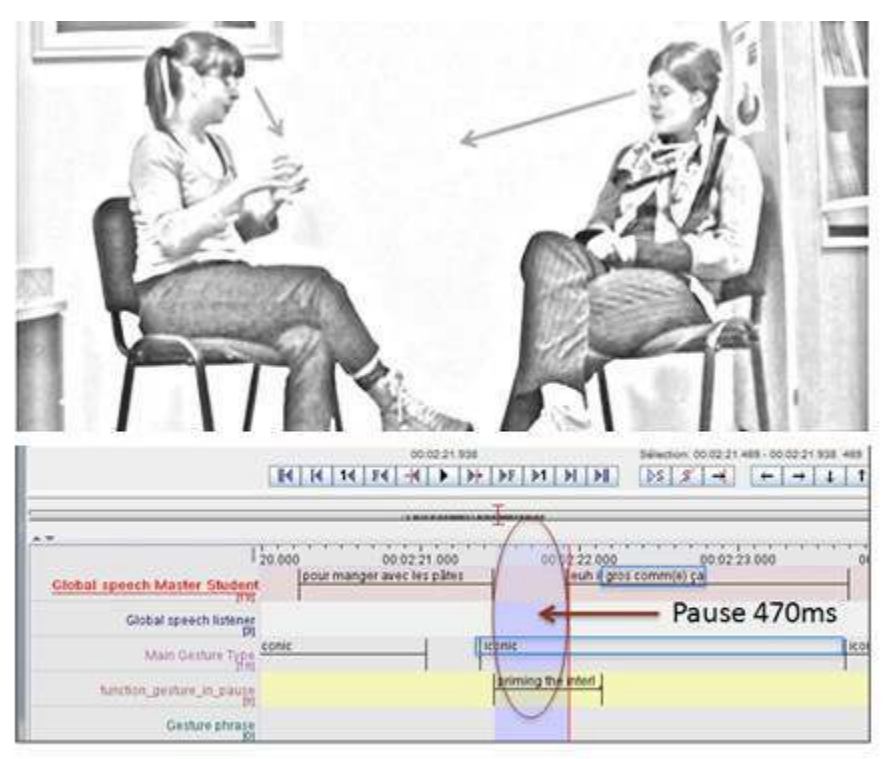

[pour manger avec les] pâtes [(0.47) euh gros comm(e) ça] synonyme ( frotter »), elle dit en parlant du fromage « pour qu'il devienne un (0.4) en $\mathrm{p}$ (e)tits fils (0.6) ». Toute cette partie depuis le début de l'énoncé jusqu'à la fin de la pause de 600ms est accompagnée d'un même geste redondant avec la parole dans lequel la main droite fait des mouvements montants et descendants tout en produisant de petits mouvements de doigts afin d'indiquer une pluie de petits morceaux de fromage (les « petits fils ») qui tombent de la râpe. L'énoncé « en p(e)tits fils » est précédé et suivi de pauses de respectivement $400 \mathrm{~ms}$ et $600 \mathrm{~ms}$ pendant lesquelles le geste se poursuit. Le motclé est donc encadré par ce geste produit dans les pauses. Cela rappelle ce que faisait Virginie en parlant des "tranches fines». Le regard de la future enseignante est clairement dirigé vers son interlocutrice. 
Figure 11 - Geste produit dans les pauses du discours - Exemple 3

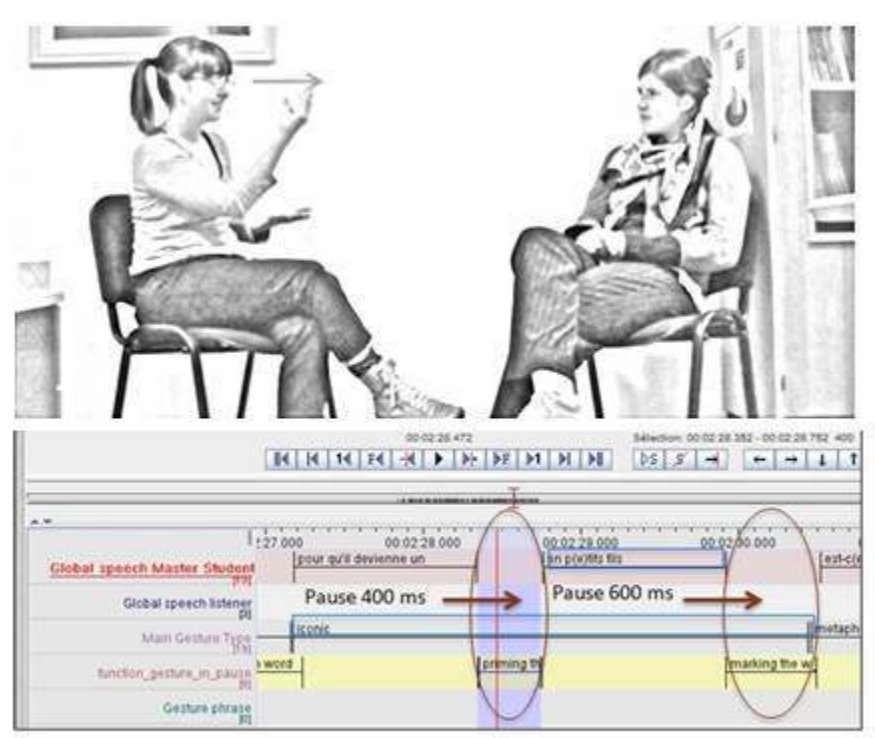

[pour qu'il devienne un (400) en p(e)tits fils (600)]

31 Comme on a pu le voir dans ces trois exemples, l'usage des pauses et la production des gestes dans ces pauses montre que l'enseignante a acquis (ou est en train d'acquérir) un discours didactique multimodal. Elle joue sur les pauses et les gestes afin de marquer les mots importants de son discours pour rendre son énoncé plus facile à comprendre. Une fois que l'étape faire comprendre est établie, Carole passe à une étape faire produire, elle dit " on dit que le fromage est » (ligne 6) et produit un geste, main ouverte, en attente d'une réponse de sa partenaire. Son geste symbolise à la fois métaphoriquement le mot manquant dans la phrase (comme un blanc à compléter dans un exercice) et une passation de tour de parole. Elle regarde également directement sa partenaire. On retrouve ici une stratégie proche de ce que faisait Virginie en demandant comment cuire la bavette. Ces différents exemples montrent que dans le corpus de classe comme dans le corpus d'explication lexicale semicontrôlé, les enseignants et futures enseignants observés utilisent conjointement geste, parole et pauses (et même aussi regard) pour faire comprendre et faire produire ${ }^{11}$. 


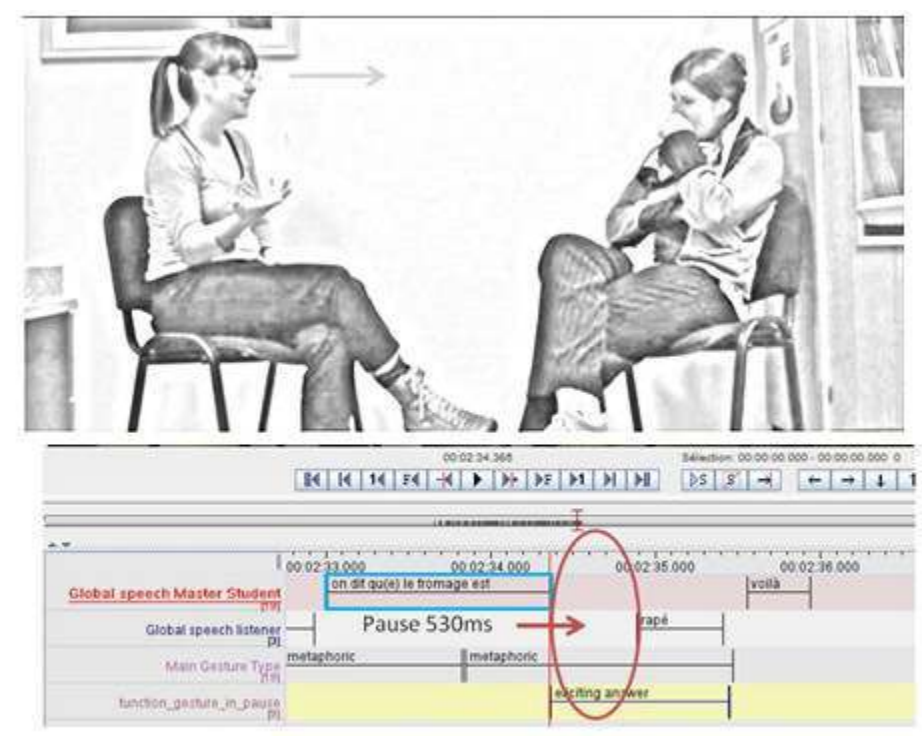

[on dit qu(e) le froma][ge est (520)]

\section{Conclusion}

Les quelques exemples détaillés ici illustrent un grand nombre de questionnements et de phénomènes. Premièrement, ils montrent que la seule prise en compte des aspects verbaux de l'interaction ne suffit pas à comprendre l'intégralité de ce qui se joue dans la classe. Deuxièmement, ils révèlent que l'agir professoral de l'enseignant est bien constitué à la fois d' " actions verbales et non verbales » comme l'avait déjà souligné Cicurel (2011 : 119) et fait écho aux propos, plus anciens, de Porcher "de même qu"il n'y a pas d'enseignant qui ne parle pas, il n'y en a aucun qui ne gesticule, à tout moment de la classe » (1989: 44). Le corps tout entier de l'enseignant participe à l'action pédagogique. La gestuelle manuelle, quant à elle, est tellement fondamentale qu'au cours de l'interaction où l'enseignante Virginie explique «la bavette", celle-ci pose les feuilles de papier qu'elle tient afin de libérer ses mains pour la suite de l'explication. Troisièmement, les exemples présentés ici montrent que la relation sémantique entre geste et parole est extrêmement solide et que les deux sont noués mais de manière beaucoup plus complexe qu'il n'y paraît à première vue. Le geste n'est pas qu'une simple illustration de ce qui est dit et n'est pas toujours un sous-titrage visuel de la parole. Il peut aussi venir désambigüiser la parole voire la compléter. Enfin, on observe dans le discours pédagogique (naturel comme provoqué) une désynchronisation de la production gestuelle et de la parole afin de mieux mettre en évidence les mots-clés du discours de l'enseignant à la fois en allongeant les pauses du discours pour mieux segmenter la parole mais également en produisant des gestes pendant ces pauses, ce qui, dans la communication « ordinaire » est beaucoup plus rare (McNeill, 1992, Stam \& Tellier, en révision). 


\section{BIBLIOGRAPHIE}

Allen, L. (2000). « Nonverbal Accommodations in Foreign Language Teacher Talk ». Applied Language Learning, vol. 11, $\mathrm{n}^{\circ} 1$. pp. 155-176.

Azaoui, B. (2014a). « Multimodalité des signes et enjeux énonciatifs en classe de FL1/FLS ». In Tellier M.\& Cadet L. (dir.) Le corps et la voix de l'enseignant : mise en contexte théorique et pratique. Paris : Éditions Maison des Langues.

Azaoui, B. (2014b). Co-construction des normes scolaires et contextes d'enseignement. Une étude multimodale de l'agir professoral. Thèse de doctorat non publiée. Montpellier: Université Paul Valéry, Montpellier III.

Barnett, M. A. (1983). « Replacing Teacher Talk with Gestures: Nonverbal communication in the Foreign Language Classroom ». Foreign Language Annals, vol. 16, n³. pp. 439-448.

Beattie, G. \& Shovelton, H. (1999). « Do iconic hand gestures really contribute anything to the semantic information conveyed by speech? An experimental investigation ». Semiotica, vol. 123, n -1/2.pp. 1-30.

Cicurel, F. (1990). « Éléments d'un rituel communicatif dans les situations d'enseignement ». In Dabène, L. ; Cicurel, F. ; Lauga-Hamid M.-C. \& Foerster, C. (dir.) Variations et rituels en classe de langue. Paris : Hatier/Didier, Crédif, coll. « LAL ».

Cicurel, F. (2011). Les interactions dans l'enseignement des langues. Agir professoral et pratiques de classe . Paris : Éditions Didier.

Cosnier, J. \& Develotte, C. (2011). « Le face-à-face en ligne, approche éthologique ». In Develotte, C., Kern, R. \& Lamy, M.-N. (dir). Décrire la conversation en ligne Le face à face distanciel. Lyon : ENS Éditions.

Dabène, L. (1984). « Pour une taxinomie des opérations métacommunicatives en classe de langue étrangère ». Études de Linguistique Appliquée, vol. 55. pp. 39-46.

Denizci, C. (2015). Utilisation des gestes coverbaux en classe de FLE. Thèse de doctorat non publiée. Istanbul (Turquie) : Université d'Istanbul.

Faraco, M. (2008). « Gestes et prosodie didactiques dans l'enseignement des structures langagières en FLE ». Actes du colloque Enseigner les structures langagières en FLE, Bruxelles, 20-22 mars 2008. [En ligne] http://gramm-fle.ulb.ac.be/95/enseigner-les-structures-langagieres-en-flebruxelles-20-22-mars-2008-actes/

Goldin-Meadow, S. (2003). Hearing gesture: how our hands help us think. Cambridge, MA: Belknap Press of Harvard University Press.

Grant, B. M. \& Grant Hennings, D. (1971). The Teacher moves. An analysis of non-verbal activity. New York : Teacher College Press, Columbia University.

Guichon, N. (2013). « Une approche sémio-didactique de l'activité de l'enseignant de langue en ligne : réflexions méthodologiques ». Education \& Didactique, vol. 7, n 1, pp. 101-116.

Gullberg, M. (2010). « Methodological reflections on gesture analysis in second language acquisition and bilingualism research ». Second Language Research, vol. 25, n 4. pp. 1-28. 
Gullberg, M., \& Holmqvist, K. (2006). « What speakers do and what listeners look at. Visual attention to gestures in human interaction live and on video ». Pragmatics and Cognition, vol. 14. pp. 53-82.

Holler, J., Shovelton, H. \& Beattie, J. (2009). « Do iconic hand gestures really contribute to the semantic information in face to face contact ». Journal of non verbal behavior, vol. 33. pp. 73-88.

Holt, B. (2014). Stratégies multimodales mises en place par les apprentis-enseignants lors d'une interaction en visioconférence : le cas des séquences d'incompréhension. Mémoire de Master 2 recherche en didactique des langues, non publié. Aix-Marseille Université.

Kellerman, S. (1992). « 'I See What You Mean': The Role of Kinesic Behaviour in Listening and Implications for Foreign and Second Language Learning ». Applied Linguistics, vol. 13, n 3. pp. 239-258.

Lazaraton, A. (2004). « Gestures and speech in the vocabulary explanations of one ESL teacher: A microanalytic inquiry ». Language Learning, vol. 54, $\mathrm{n}^{\circ} 1$. pp. 79-117.

Mackey, A., Al-Khalil, M., Atanassova, G., Hama, M., Logan-Terry, A. \& Nakatsukasa, K. (2007). « Teachers'intentions and learners' perceptions about corrective feedback in the L2 classroom ». Innovations in Language Learning and Teaching, vol. \&, $\mathrm{n}^{\circ}$ 1. pp. 129-152.

Martina, C. (1991). «Eléments d'une méthode corrective en classe d'anglais : la gestuelle ». Les langues modernes, vol. 85, $\mathrm{n}^{\circ} 1$. pp. 45-49.

McCafferty, S. G. \& Stam, G. (Dir.). (2008). Gesture: Second language acquisition and classroom research. New York: Routledge.

Mcneill, D. (1985). « So you think gestures are nonverbal? ». Psychological Review, vol. 92, n 3. pp. 350-371.

Mcneill, D. (1992). Hand and Mind: What gestures reveal about thought. Chicago: The University of Chicago Press.

Mcneill, D. (2005). Gesture \& thought. Chicago: The University of Chicago Press.

Mondada, L. (2012). « Organisation multimodale de la parole-en-interaction : pratiques incarnées d'introduction des référents ». Langue française, vol. 175, n 3. pp. 129-147.

Özyürek, A. (2002). « Do Speakers Design Their Cospeech Gestures for Their Addressees? The Effects of Addressee Location on Representational Gestures ». Journal of Memory and Language, vol. $46, \mathrm{n}^{\circ}$ 4. pp. $688-704$.

Pavelin, B. (2002). Le geste à la parole. Toulouse : Presses Universitaires du Mirail.

Porcher, L. (1989). « Didactique : Pour la beauté du geste ». In Calbris, G. \& Porcher, L. (dir). Geste et communication. Paris : Didier, Coll. LAL.

Rauscher, F. H., Krauss, R. M. \& Chen, Y. (1996). « Gesture, speech, and lexical access: the role of lexical movements in speech production ». Psychological Science, vol. 7, pp. 226-230.

Stam, G. \& Tellier, M. (en révision). « The sound of silence: how gestures in pauses can enhance interaction ». In R. B. Church, M. W. Alibali and S. D. Kelly (dir.), Why Gesture? How the hands function in speaking, thinking and communicating. Amsterdam, NL: John Benjamins.

Tellier, M. \& Cadet, L. (dir.) Le corps et la voix de l'enseignant : théorie et pratique (pp. 7-12). Paris : Éditions Maison des Langues, pp. 7-12. 
Tellier, M. \& Stam, G. (2012). « Stratégies verbales et gestuelles dans l'explication lexicale d'un verbe d'action ». In Rivière, V. (2012). Spécificités et diversité des interactions didactiques. Paris : Riveneuve éditions.

Tellier, M. (2008). « Dire avec des gestes ». In Chnane-Davin, F. \& Cuq, J.P. (dir) Du discours de l'enseignant aux pratiques de l'apprenant en classe de français langue étrangère, seconde et maternelle. Le Français dans le monde, recherche et application, vol. 44, pp. 40-50.

\section{NOTES}

1. Dans la transcription des interactions verbales, les chiffres donnés entre parenthèses correspondent à la durée des pauses du discours et sont donnés en millisecondes. Les parties soulignées montrent les chevauchements dans les tours de parole et les XXX indiquent des séquences incompréhensibles. Les parties de mots entre parenthèses montrent les phonèmes omis. Tous les prénoms des participants ont été changés.

2. Dans les lignes qui suivent nous nous focaliserons sur quelques éléments gestuels pour ne pas trop alourdir le texte d'exemples et de captures d'écran. Nous espérons que les quelques extraits choisis montreront la richesse multimodale de la séquence.

3. L'usage du terme kinésique permet ici d'englober non seulement les gestes manuels mais aussi les mimiques faciales, le regard et les postures.

4. Les parties entre crochets indiquent le moment de la phrase où apparait un geste.

5. Gestes battant la mesure souvent de bas en haut et qui peuvent varier dans l'amplitude.

6. Un hold gestuel est une pause dans la production du geste, durant laquelle il reste en suspens avant de poursuivre sa course.

7. Le logiciel Elan (Sloetjes \& Wittenburg, 2008) permet de retranscrire facilement les différentes modalités qui cooccurrent dans le discours grâce à un système de partition. On peut donc lire à la fois sur un axe syntagmatique la chronologie de la parole verbale et des différentes productions kinésiques et sur un axe paradigmatique, la synchronisation de production de ces différents éléments. A noter sur les images, on voit souvent d'autres personnes en arrière-plan, il s'agit des autres futurs enseignants chargés de co-animer la séance puisque celle-ci était préparée en groupe dans le cadre de leur formation en master.

8. Geste abstrait représentant un concept, en général, les mains semblent contenir une entité invisible.

9. Geste conventionnalisé et marqué culturellement comme l'index tirant la paupière inférieure vers le bas pour signifier "mon œil". Ces gestes peuvent être apparentés à des expressions idiomatiques.

10. Les chiffres entre parenthèses comme (330) indiquent la durée des pauses en millisecondes. Les parties entre crochets montrent les éléments du discours pendant lesquels des gestes apparaissent, par exemple: [petit morceau de bœuf]. Celles qui sont en gras et accompagnées d"un numéro -1- renvoient aux exemples illustrés ci-dessous avec des photographies.

11. L'usage des gestes dans les pauses existe aussi avec les partenaires natifs mais dans des proportions bien moindres (voir Stam \& Tellier, en révision). 


\section{RÉSUMÉS}

Dans cet article, nous analyserons comment les modalités kinésiques (gestes des mains, regards, mouvements de tête, mimiques faciales) s'articulent avec la parole de l'enseignant pour construire le discours pédagogique. Dans un premier temps, nous reprendrons les trois fonctions pédagogiques de Dabène (1984) en montrant comment elles se réalisent multimodalement. Dans une seconde partie, nous considèrerons les liens sémantiques entre le geste et la parole et enfin, dans une troisième partie, nous procèderons à une analyse détaillée de la synchronie entre le geste et la parole.

In this paper, we analyze how the kinesthetic modalities (hand gestures, gaze, head movements, facial expressions) are articulated with teacher speech to build pedagogical discourse. We first use the three pedagogical functions defined by Dabène (1984) showing how they are realized through various modalities. In the second part, we consider the semantic links between gesture and speech and finally, we conduct a detailed analysis of synchrony between gesture and speech.

\section{INDEX}

Mots-clés : geste pédagogique, agir professoral, multimodalité, pauses du discours

Keywords : teaching gestures, teaching action, multimodality, speech pauses

\section{AUTEUR}

\section{MARION TELLIER}

